\title{
Polysèmes
}

Revue d'études intertextuelles et intermédiales

\section{Histoires de fantômes : insoutenables légèretés et ombres portées de l'enfant dans le roman britannique contemporain}

The unbearable lightness of the child in contemporary British fiction: from ghost stories to monstrous tales

\section{Camille François}

\section{OpenEdition}

Journals

Édition électronique

URL : http://journals.openedition.org/polysemes/390

DOI : $10.4000 /$ polysemes.390

ISSN : 2496-4212

Éditeur

SAIT

\section{Référence électronique}

Camille François, «Histoires de fantômes : insoutenables légèretés et ombres portées de l'enfant dans le roman britannique contemporain », Polysèmes [En ligne], 13 | 2015, mis en ligne le 14 octobre 2015, consulté le 20 avril 2019. URL : http://journals.openedition.org/polysemes/390 ; DOI : 10.4000/ polysemes.390

Ce document a été généré automatiquement le 20 avril 2019

Polysèmes 


\section{Histoires de fantômes : insoutenables légèretés et ombres portées de l'enfant dans le roman britannique contemporain}

The unbearable lightness of the child in contemporary British fiction: from ghost stories to monstrous tales

Camille François

1 Commentant Freud et Rousseau, Catherine Clément et Hélène Cixous écrivent dans La Jeune Née : "L'enfant est le miroir de l'adulte ; miroir-fée qui donne la bonne réponse ${ }^{1}$ . L'enfant comme miroir, n'intéressant pas en soi mais comme prisme signifiant et moyen d'une vérité sur son autre, l'adulte, est à rapprocher du motif de l'objet transparent présent dans plusieurs romans contemporains de l'enfance. On peut penser à la loupe dans When We Were Orphans de Kazuo Ishiguro, quête des origines qui prend la forme d'un enquête policière ; mais aussi, outre-Atlantique, à Cat's Eye, dans lequel Margaret Atwood figure la redécouverte de l'enfance par cette petite bille transparente fétiche des enfants. Si certains auteurs contemporains ressentent le besoin de métaphoriser l'enfance par ces objets de verre, c'est que leurs textes sont hantés par un héritage poétique de l'écriture de l'enfant comme transparence, que tout ensemble ils exhibent et esquivent. Historiquement, cette transparence est à comprendre comme une aliénation poétique, comme la spectralité d'un sujet enfant réduit par les textes au simple rôle de miroir donc, de loupe, ou d'œil, donnant accès au "réalisme " illusoire d'une immédiateté qui contournerait l'art. C'est contre cette transparence fortement inspirée de Rousseau ${ }^{2}$ qu'une génération de romanciers britanniques semble écrire aujourd'hui, comme le montre le détournement de l'objet transparent chez Ishiguro :

[...] the package had been wrapped in numerous sheets, and my friends would laugh noisily each time I removed one layer, only to be confronted by another. [...] What I 
did eventually uncover was a weathered leather case, and when I undid the tiny

catch and raised the lid, a magnifying glass. ${ }^{3}$

When We Were Orphans s'ouvre sur le dévoilement progressif d'un objet transparent soigneusement empaqueté, le suspense syntaxique produisant comme une fin en soi ce que l'on associe plus traditionnellement au moyen de trouver cette fin - tout comme d'ailleurs, chez Atwood, l'œil de chat n'est pas le prisme au travers duquel on pourrait espérer voir l'enfance, mais une manière de renvoyer le lecteur à son propre œil. Ce qu'illustre cet épisode, c'est l'importance du filtre, déviant aujourd'hui le regard curieux d'une profondeur derrière la vitre, pour l'arrêter à cette surface elle-même porteuse de sens. Dès lors, il faudra se demander quelles conséquences éthico-esthétiques de cette traditionnelle écriture de l'enfance sont mises en évidence - et au pilori - par les textes postmodernes, et quelle nouvelle définition de la transparence, ou quelle opacité, est proposée en échange. Se pose aussi la question de comment arrêter le regard sur l'enfant : toute pigmentation ne risque-t-elle pas d'équivaloir à une souillure, cet autre écueil traditionnel de l'écriture de l'enfance?

\section{Enfantômes, enfantasmes}

2 Historiquement, l'enfant tient lieu en littérature de fenêtre à l'artiste : crédité depuis Rousseau de cette pureté de langage seule capable de transmettre une vérité du monde ${ }^{4}$, il garantit par son peu d'être qu'aucune subjectivité parasite ne vient altérer l'image perçue. On aura reconnu le modèle de l'enfant focalizer mis à l'honneur par James, qui cherche avec Maisie ${ }^{5}$ à transmettre ce qu'un point de vue plus mûr aurait risqué d' adultérer. "The clear eye of the child is not dimmed by the hazier and more mediated vision of the adult $\|^{6}$, lit-on aujourd'hui encore sous la plume d'une critique, preuve s'il en est que le mythe de l'enfant garant de la mimesis a la vie dure. Comme le fait remarquer Reinhard Kuhn dans Corruption in Paradise: The Image of Childhood in Western Literature $^{7}$, le phénomène est d'autant plus dérangeant que, transparence du monde, il est opacité profonde de l'enfance. Si What Maisie Knew se clôt sur des mots qui soulignent la potentialité ironique du titre ( Mrs Wix gave a sidelong look. She still had room for wonder at what Maisie knew. $\left.»^{8}\right)$, c'est que, dans ce texte, le regard du lecteur ne s'arrête pas à l'enfant, mais le pénètre et le dépasse, de sorte que, tout au fait des agissements des adultes que nous sommes, nous serions, à l'instar de Mrs Wix, bien en peine de prétendre connaître vraiment cet angle mort du récit qu'est Maisie. Prolongement des fantasmes romantiques de transparence, les dérives du discours freudien dénoncées par Virginia Blum dans Hide and Seek: The Child Between Psychoanalysis and Fiction", associent inlassablement l'enfant à l'idée de vérité, de "miroir fée qui donne la bonne réponse » pour reprendre la citation de La Jeune Née: immatériel là encore, il prend la forme de «l'enfant en nous », cette clef de l'être cherchée en transparence d'un adulte spectral et ne permettant pas davantage d'approcher un quelconque enfant sujet du texte ${ }^{10}$. Cet autre fantasme d'enfant-vérité est implicitement mis à mal dans le texte d'Ishiguro, que seule habite une pauvre âme errante, soucieuse jusqu'à l'obsession de remonter le fil du temps afin de comprendre, croit-elle, qui elle est vraiment. When We Were Orphans affiche en réalité la nature illusoire de cette quête rétrospective du moi comme trésor perdu, menant à la négligence d'un présent et d'une maturité fantomatiques, éclipsées par l'enfance absente. 
3 Nous parlons de fantômes, d'« enfantômes » pourrait-on dire: le mot-valise s'impose naturellement lorsque l'on sait que la garantie de cette vérité du récit permise par l'enfant, c'est son absence de qualité. Par définition infans, du latin in fari, celui qui n'a pas de langage ${ }^{11}$, il est aussi innocent, immaculé, ignorant, imbécile (au sens de faible) autant de préfixes négatifs qui le mettent au cœur du texte du fait, précisément, de son absence d'être. Pas étonnant donc que depuis Peter $\operatorname{Pan}^{12}$, les écrivains se passent l'un l'autre le motif de l'ombre perdue comme moyen de mettre au jour la nature d'âmes damnées de leurs personnages. Pur œil, ou image de la vérité du sujet adulte qui ne permet finalement qu'un rapport narcissique de soi à soi ${ }^{13}$, l'enfant est cet éternel Peter Pan dont le corps trop léger s'envole pour un Neverneverland qui fait office de purgatoire. Dans The Children's Book, roman dont le titre indique qu'A.S. Byatt s'efforce de redonner à l'enfant la place qui lui est due, le jeune Tom est fasciné par les histoires qu'écrit pour lui sa mère, auteur de livres pour enfants, et qui le mettent en scène en "shadowless hero ", en quête de son ombre ${ }^{14}$ - il finira par se suicider, trop conscient de son immatérialité après s'être reconnu dans la pièce de James Barrie. Diaphane, cet enfant du passé mis en scène par le récit historique de Byatt, rappelle que Rousseau défendait dans l'Émile une éducation "négative ", qui constituerait un retranchement de qualités plutôt qu'un développement de potentialités ${ }^{15}$. Parfait prisme fictionnel pour sa mère dès lors, Tom souffre de l'insoutenable légèreté d'un être condamné à une absence de corps qui serait aussi une absence d'être, comme l'illustre cet échange avec sa sœur vers la fin du récit : "'Sometimes, Dorothy, I wake up and think I'm not there. [...] I'd like to be able to vanish into the hedge, like one of those things you can't see, if they don't move'. [...] He wrinkled his brow. His brows, Dorothy thought, were fair and innocent. What was it that wasn't there? $»^{16}$. Cette condition spectrale d'enfants vidés par le littéraire est aussi le point de départ de The Child in Time de Ian McEwan : un écrivain pour enfants - encore un - voit sa fille de trois ans disparaître sans que l'on n'apprenne jamais rien d'un supposé kidnapping qui prend de ce fait une valeur allégorique. « He was the father of an invisible child $»^{17}$ constate le narrateur à propos de Stephen, avant que son histoire de fantômes ne glisse véritablement dans le fantastique, et qu'il ne se retrouve lui-même dans la position des enfants qu'il écrivait. Pur esprit, il assiste dans une scène hallucinatoire à la décision de ses parents de ne pas avorter de lui : «Had the couple glanced up and to their left, towards the window by the door, they might have seen a phantom beyond the spotted glass [...] $\aleph^{18}$. Dans ces nouveaux récits, le personnage écrivain renvoyé à son tour de l'autre côté de la vitre et affecté par sa transparence, est donc mis en demeure de prendre la mesure de la condition imposée à l'enfant par les textes traditionnels, en en faisant luimême l'expérience, ou en devant subir sa disparition.

4 L'aliénation poétique qui accompagne cette transparence masque également une violence sémiotique : James Kincaid, dans un ouvrage provocateur intitulé Child-loving: the Erotic Child and Victorian Culture ${ }^{19}$, explique comment la pureté et le peu d'être de l'enfant sont précisément ce qui en fait un objet érotique. L'« enfantôme » débouche en quelque sorte sur l'« enfantasme ", puisque, signifiant libre, l'enfant est le parfait réceptacle de toutes les valeurs en mal d'incarnation. S'il ne veut rien dire, mais laisse irrémédiablement voir au travers de son joli teint de porcelaine, il constitue une puissance symbolique qui permet de suggérer une chose et son contraire. Signe blanc, il perd son épaisseur de personnage dans le feuilletage des sens qui lui sont associés, entre inquiétante étrangeté et symbole du moi le plus profond, conservatisme et renouveau, innocence originelle et souillure indélébile, pour n'en citer que quelques-uns. Dans London Fields, Martin Amis 
renvoie ses lecteurs à l'absurdité de la belle transparence de l'enfant, qui en fait tout à la fois un sujet désirable et creux, un être spécieux aux deux sens du terme. Proposant en guise de narrateur la réécriture badine d'un Humbert Humbert épris d'une Lolita de quelques mois seulement ${ }^{20}$, il décrit à l'aide d'objets transparents le regard vitreux de la petite Kim, dont la beauté s'explique par son pouvoir de suggestion, sa capacité à accueillir l'univers tout entier: "Kim's eyes make me understand. Jewellery, precious minerals, intricate glassware, dead beauty, it's all fine: an attempt to summon the living galaxy of a baby's eyes. The baby's sparklers, the Milky Way of babies... $»^{21}$. Byatt souligne davantage encore le lien dérangeant entre transparence et child-loving, puisque l'instrumentalisation de l'enfant à des fins artistiques transforme dans The Children's Book l'acte créatif en viol, la transparence en risque de pénétration. L'employée de maison du potier Benedict Fludd découvre ainsi, sans mauvais jeu de mot, le pot aux roses sous la forme de représentations obscènes des deux filles de l'artiste, Imogen et Pomona, par leur père :

On the shelves were pots. [...] most were small, and glimmered white in the shadows, white-glazed china, unglazed biscuit. [...] The pots were obscene chimaeras, half vessels, half human. They had a purity and clarity of line, and were contorted into every shape of human sexual display and congress. [...] Some of them had Imogen's long face and drooping shoulders: some of them were plump Pomona. The males were faceless fantasms. [...] the open mouths and clutching hands were not all the same age, went back years, into childishness. ${ }^{22}$

On voit ici comme l'ekphrasis, dans son emphase sur la corrélation entre pureté, blancheur de l'objet et contorsions, perversion de l'acte incestueux, explique la nature fantomatique des filles de l'artiste, potiches littérales, récipients des fantasmes du père.

\section{Chiaroscuro du roman contemporain : nouvelles transparences et ombres portées}

Rendre à Peter son ombre, opacifier la potiche, griser voire noircir l'enfance : c'est dès lors l'idéal poétique tout en nuances du chiaroscuro que plusieurs romanciers contemporains substituent à une illusoire transparence comme accès à la "vérité » du monde et du sujet, à la valeur. Il consiste par exemple à suspendre les jeux de signification du signe blanc pour produire l'enfant en énigme, décrit ainsi par Reinhard Kuhn : «[... ] he is the forever undecodable signifier. His appearance is transparent, but in its inexplicability forever opaque. The child as an enigma that no amount of speculation can resolve is a recurrent theme in contemporary literature $»^{23}$. L'intervention de l'intrigue policière chez Ishiguro illustre bien cela, puisque When We Were Orphans prend le temps de construire une figure exemplaire du détective dont l'enquête la plus ardue, et qui le mènera presque à cette impasse du sens qu'est la folie, est celle de son enfance. La présence de l'enfant-énigme transforme également les textes en récits gothiques, à l'image de The Fifth Child de Doris Lessing, dans lequel une mère éperdue confesse tout ensemble son incapacité à comprendre Ben, ce mystérieux cinquième enfant, et la peur qu'il lui inspire :

From the high skylight fell a distorted rectangle of light, and in it stood Ben, staring up at dim sunlight. She could not make out what he wanted, what he felt... [...] in one leap he had reached the dark at the edges of the eaves and vanished. All she could see was the obscurities of an attic that seemed boundless. [...] She was rigid with terror. ${ }^{24}$ 
6

Nouveau « continent noir ", comme le montrent les jeux d'ombre et de lumière dans cet extrait, l'enfant échappe à la transparence par une incursion dans l'horreur, en déjouant les enquêtes, ou en bloquant les traditionnelles interprétations freudiennes qui le faisaient clef du sujet et du sens. Dans London Fields par exemple, si Marmaduke a fait de la castration (fort peu symbolique au demeurant) de son père Guy son activité principale, le texte d'Amis qui force la caricature de l'ÆEdipe jusqu'au grotesque nous refuse pourtant cette trop simple analyse, comme elle est refusée aux parents, Guy et Hope, après consultation d'un spécialiste :

'On the hostility-to-you question,' said Hope, 'I thought he made a change from Freud.'

'So did I. But I prefer Freud. I'd rather Marmaduke didn't like me for Freudian reasons. I don't like him not liking me because he just doesn't like me. Why shouldn't he like me? I'm incredibly nice to him all the time. ${ }^{25}$

Comme souvent chez Lessing, la supposée explication se retourne ici en question, et l'enfant laisse planer sur le texte l'ombre de son inquiétante étrangeté.

7 Autre contournement de la transparence érotisée, le rôle poétique essentiel qu'accordent Peter Ackroyd et A.S. Byatt à l'intertextualité permet de démultiplier le miroir qu'était traditionnellement l'enfant en une galerie des glaces dont la surface vitrée ne se traverse plus, mais suppose une attention soutenue à un reflet vertigineusement présent. Chez eux en effet, l'enfant est une image répétée à l'infini, dont la seule vérité est celle décrite par Barthes dans $S / Z^{26}$, par Riffaterre ${ }^{27}$ aussi dans son étude de la relation entre intertexte et mimesis : que l'enfant du texte ne transcrit pas un référent, mais qu'il est, comme le montrent les recours à l'ekphrasis ou à l'allusion intertextuelle, toujours la représentation d'une représentation. Sujet de pièces de théâtre, de contes et de poteries chez Byatt, de textes canoniques et de tableaux chez Ackroyd, les deux jeunes protagonistes de The Children's Book et de English Music traversent leurs intrigues respectives sur un double plan: celui du récit principal, mais aussi celui des histoires enchâssées, dont les conventions comme l'italique ou le passage à la troisième personne accentuent la nature fictionnelle. Chez Ackroyd, le jeune Tim est successivement projeté dans Alice in Wonderland, Great Expectations, Robinson Crusoe, découvrant au fil des textes sa propre nature fictionnelle et le sens du mot « palimpseste » évoqué par son père au début $\mathrm{du}$ récit $^{28}$. Lors d'un de ces voyages dans le texte, Charles Dickens lui explique métaphoriquement qu'il n'aurait pas pu exister sans Pip, sans Oliver, sans David et les autres $^{29}$, dont il n'est que la répétition, la continuation: "Houses are always built on, Timothy. One gives place to another. Foundations laid upon foundations. The end of one is the beginning of another $»^{30}$. Les hantises de ce que Harold Bloom a appelé «the anxiety of influence ${ }^{31}$, dont les éternels retours contraignent le roman contemporain à la post-représentation, à la coexistence des modèles, conduit à l'émergence d'une nouvelle transparence, un réalisme de l'art au sens moderne cette fois, montrant l'artifice pour ce qu'il est, laissant voir les ficelles de la marionnette enfant, plutôt que cédant, dans les mots de Riffaterre aux « sirènes de l'illusion référentielle " ${ }^{32}$. Paradoxalement, ce discours confus du leurre débouche donc sur une forme de vérité, sur la nature du matériau fictionnel, mais aussi sur la complexité du vivant, diffracté, multiple, à l'image de l'enfance des personnages.

8 Il n'est sans doute pas anodin que deux des ouvrages cités, London Fields et The Child in $T_{i m e}{ }^{33}$, mentionnent le principe d'incertitude d'Heisenberg, qui, en physique, décrit la perturbation involontaire mais inévitable de l'observateur sur son objet du simple fait 
qu'il l'observe, faussant nécessairement l'expérience. L'intérêt de la fiction pour cette idée se retrouve dans la volonté de tirer parti de l'enfant non plus comme prisme transparent, mais comme filtre, de préférer les ressources poétiques de la médiation au fantasme de l'immédiateté. Si l'enfant continue de nous transmettre le monde, on n'attend plus la restitution parfaite d'un regard cristallin, mais un subtil jeu de différences qui donnent à lire, en transparence du portrait faussé du monde, un portrait "vrai » de celui qui le regarde. Jean Pouillon a analysé cet aspect de la "vision avec" dans Temps et roman, montrant comment ce qui semble un portrait par détour nous amène en réalité au plus près du personnage - de l'enfant en l'occurrence : «il est vu [...] dans l'image qu'il se fait des autres, en quelque sorte en transparence de cette image. En somme nous le saisissons comme nous nous saisissons nous-mêmes $»^{34}$. Dans Atonement, roman qui s'intéresse aux conséquences tragiques de la mauvaise lecture de scènes intimes par la jeune Briony ${ }^{35}$, la confrontation de la vision de l'enfant à d'autres par l'alternance du point de vue en dit long sur son incapacité fondamentale à saisir le réel. Toutefois, cette déformation n'est plus considérée comme un échec poétique, mais comme le moyen d'une réflexion herméneutique sur la possibilité ou les dangers de l'interprétation. Floutant l'accès au sens par leur regard, par leur langage, les enfants du clair-obscur permettent aussi une forme de jouissance qui ne passe plus par leur aliénation dans le texte mais par l'acte de lecture: "[i]ndeterminacy elicits participation » résume Ihab Hassan ${ }^{36}$, récitant les tables de la loi d'un postmodernisme qui met à l'honneur un langage-obstacle opposé en tout à la langue rêvée par Rousseau. Selon Marcel Cornis-Pop dans Mimesis, Semiosis and Power, l'objectif en est de bloquer les réflexes interprétatifs stéréotypés du lecteur qui conduisent toujours à la production du même sens par les mêmes mots ${ }^{37}$ - une tendance souvent poussée à l'extrême chez Amis par l'entremise du babil, qu'il faut parfois interpréter contre les traductions douteuses qu'en font les adultes du texte. Dans la scène suivante, Guy substitue aux mots de son fils ceux d'un abécédaire conforme, sans se rendre compte que le babillage de l'enfant a un sens en l'état, dont le lecteur est le garant : il décrit le monde que son père lui a laissé, un monde bien différent de celui des livres d'enfants.

'Come on. And read a book. [...] What's that darling?'

'Dick.'

'Stick. Very good. Ssssstick. Now here's a flower. Say 'flower'... Those are the petals.

And this bit down here is -'

'Dork.'

'Very good, darling. Excellent. Now what do you call this? Where the tree used to be.

Like in our garden. Where they've chopped it down.'

'Dump. ${ }^{.38}$

Dans le jeu qui substitue dork à stalk et dump à stump, le mot absent et le sens instable réveillent l'exégète en nous, favorisent une poétique de l'à peine dit plutôt que de l'élucidation systématique, une juxtaposition du clair et de l'obscur qui instaure la collaboration poétique à l'endroit de l'enfant-filtre.

\section{Figurines de porcelaine : faux et surfaces sales}

Ceci étant dit, il semble que parfois la tentative de sauver l'enfant de sa délétère transparence conduise certains auteurs à forcer le trait, confondant opacité et souillure, à montrer voire à monstrer trop, puisque c'est de là que vient étymologiquement le monstre ; à se cantonner aussi dans l'impasse postmoderne d'une exhibition de la surface qui manque de basculer dans la superficialité. Cherchant à se défaire des caprices du 
mimétisme, plusieurs écrivains britanniques contemporains lui ont ainsi substitué le grotesque, voire le pornographique, comme mode d'écriture de l'enfance qui permette de contourner l'érotique. Ne plus utiliser l'enfant comme voile affriolant, mais le remettre au centre, et le montrer tout entier, nu, et laid, les fluides qui le recouvrent ad nauseam chez Amis par exemple assurant qu'il ne peut plus être transparent :

As [Guy] was dragging him towards the front door and reaching out to free the doublelock, Marmaduke 'went supervoid', in the local phrase [...] The child, in other words, had swamped himself in ordure. When Guy unravelled Marmaduke's scarf he saw that some of it was even peeping over the collar of his shirt. In the nursery again Guy [...] hosed Marmaduke down in the master bathroom, and wrestled him back into nappy-liner, nappy, nappy pants, vest, shirt [...] This time, he actually got the front door open before Marmaduke was noisily and copiously sick. ${ }^{39}$

La dimension comique procure encore ici la sécurité de la distance et rappelle que ce mode d'écriture pornographique du tout-visible ${ }^{40}$ est autant une parodie des représentations médiatiques contemporaines, que l'inversion carnavalesque d'une tradition littéraire. Ainsi, c'est à présent le trop de corps de l'enfant qui réduit celui des adultes alentours à la transparence :

Now here came Marmaduke. Defeatedly watched by the stunned au pair (her presence diluted to a mere reflection in the glass), the little boy erupted through the double doors. Although Guy and Hope responded with grooved swiftness, Marmaduke harpooned himself face first into the table leg [...] Then the world rocked: broken glasses, chipped china, childblood, spilt milk, spilt milk. ${ }^{41}$

Comme le montre cet extrait, tous s'effacent devant le personnage pourtant secondaire de l'enfant qui occupe ici la totalité du visible, brisant les miroirs, offrant l'intérieur de son corps à tous les regards. Chez Lessing (comme d'ailleurs pour les corps incestueux des enfants de McEwan dans The Cement Garden ${ }^{42}$ ), le ton n'est pas le même, et l'on s'éloigne de la légèreté rabelaisienne du grotesque, l'objectif étant de rappeler sa responsabilité éthique à l'adulte en le confrontant à un spectacle sordide. Comme l'explique Madelena Gonzalez dans "The Aesthetics of Post-Realism and the Obscenification of Everyday Life » : « the readers [...] are obliged to confront themselves in the act of confronting the horror. Like spectators at an interactive peepshow, they are no longer merely observers but profoundly and morally implicated by the very performance of watching in which they are engaged $»^{43}$. C'est bien ce qui se joue dans The Fifth Child, l'horreur des corps jetés sous les yeux du lecteur servant d'interpellation violente, interdisant toute complaisance :

In the cots were - monsters. [...] A baby like a comma, great lolling head on a stalk of a body [...] a small girl all blurred, her flesh guttering and melting - a doll with chalky swollen limbs [...] A child seemed at first glance normal, but then Harriet saw there was no back to its head; it was all face, which seemed to scream at her. ${ }^{44}$

Décrire ce qui est (en l'exagérant, certes), plutôt que rêver à ce qui doit être : ces textes rendent obsolète l'association entre déni coupable du réel et écriture de l'enfance, dénoncée par F.R. Leavis en ces termes: «[a] theme that so obviously lends itself to nostalgia, sentimentality, false idealism and drug-addictive evasion - to diverse forms of sick refusal to recognize unequivocally, not merely the actual, but the real $»^{45}$.

Mais d'autres représentations que le monstre affleurent dans ces écritures nouvelles de l'enfance, qui signalent l'importance de l'attention prêtée à la surface, à travers notamment le motif de la contrefaçon cher à Byatt, dont le texte s'ouvre dans un musée par une double leçon d'authentification: Philip, le garçon mystérieux qui se cache dans le V \& A, est découvert par Tom « framed between fake basalt and fake obsidian $»^{46}$, tandis que la mère de ce dernier écoute le conservateur discourir sur les faux qu'il collectionne ${ }^{47}$ 
. Se manifestant également à travers la figure du clone dans Never Let Me Go de Ishiguro ${ }^{48}$ et de l'imposteur dans The Child in Time, ce motif semble signaler que l'enfant sous ses diverses formes est interchangeable, mais surtout, que l'original est perdu, que le deuil doit être fait d'une quelconque essence de l'enfant. Nous ne lisons ainsi rien des modèles plus « réels » dont les enfants clonés de Hailsham ne sont que la copie dans Never Let Me Go - pas plus que l'on ne nous donne d'entrée de jeu les moyens de deviner que les enfants rencontrés par Stephen dans The Child in Time, au cours de sa quête du véritable enfant perdu, ne sont que des fantoches : Ruth, cette petite fille en laquelle il croit reconnaître la sienne, comme Charles, son ami travesti en petit garçon, font au premier abord parfaitement illusion. C'est contre cette fâcheuse tendance de la copie à se faire passer pour l'original que London Fields nous met en garde, soulignant l'importance d'identifier ces images pour ce qu'elles sont : des simulacres que la culture actuelle et l'invasion des médias voudraient nous faire prendre pour authentiques. L'utilisation du terme «live » dans l'extrait qui suit et la nuance apportée à l'adjectif "present» signalent ainsi l'opposition floue entre un modèle qui a perdu sa qualité originelle, et une représentation démultipliée :

But they were not alone. Marmaduke was present, in electronic form [...] in the months after Marmaduke was born, [Guy and his wife had] gather[ed] footage of Marmaduke screaming his head off in the playpen, Marmaduke screaming his head off in the park, Marmaduke screaming his head off in the swimming-pool. They soon stopped bothering. After all, there was so little difference between the home movie and the closed-circuit monitor which gave them Marmaduke screaming his head off twenty-three hours a day as it was. And when the twin screens weren't giving it to them [...], Marmaduke was giving it to them: live. ${ }^{49}$

11 Si l'on n'écrit plus par rapport à une profondeur, mais, comme l'atteste la mode des textes-palimpsestes, en regard des surfaces envahissantes du passé qui rendent toute écriture moderne de seconde main, alors on écrit moins l'enfance que les signes de l'enfance, substituant une relation horizontale - de la profondeur de l'essence à la surface des formes existentielles - à une relation verticale qui consiste en une juxtaposition, une répétition de représentations interchangeables en deux dimensions. À propos de cette préférence de l'art postmoderne, Hassan écrit : «Its hard, flat surfaces repel mimesis " ${ }^{50}$. C'est le choix de Martin Amis, la présence de Marmaduke dans le texte se faisant sur le mode du gag de la castration ou du gag scatologique répété à l'envi, plutôt que de l'approfondissement d'un personnage crédible, qui consisterait à lui donner une rondeur autre que celle des couches de peinture fécale qui y sont une à une appliquées. Ce que certains dénoncent alors comme la longue danse mortifère des simulacres caractéristique de la postmodernité telle que l'a décrite Baudrillard ${ }^{51}$, d'autres voient comme une possible libération du cantonnement de l'enfance à l'expression de valeurs qui lui sont artificiellement associées. Car Baudrillard explique aussi que l'arrêt sur image qui accompagne cette sérialisation, par opposition à la quête d'une profondeur-vérité, bloque le processus interprétatif qui mène à l'idéologie ${ }^{52}$ - un point que Byatt inscrit au cœur de son texte par l'intermédiaire du personnage de Gabriel. Celui-ci raconte les ravages de la psychanalyse dont il a été le jouet, et qui, dans sa tentative de lui fixer un sens, de faire de lui un objet en trois dimensions, a manqué de le détruire : « No man has a right to dictate another man's inner life [...] They had invented me, do you see? [...] They had made me into a - into an unpleasant sculpture, or painting $"^{53}$. Il rappelle alors l'importance de l'attention prêtée à la surface par une ekphrasis salvatrice - la clef étant la conscience de cette surface comme surface, qui ne doit pas être prise pour une profondeur : 
'[...] it was a dream which showed me the way out. [...] I dreamed I was in a studio full of light. I was surrounded by canvases perfectly painted. They were all very pale . White on white, with minimal shadows, in full light. Vast paintings, of a cup with its saucer and a silver spoon, on an endless white starched cloth, with folds in it. [...] I analysed it for myself. It said to me - like a commandment - consider the surfaces.

Care for the surfaces. Don't dig under. ${ }^{54}$

Si creuser pour chercher un sens en transparence de la forme donnée équivaut pour Gabriel à creuser sa tombe, plusieurs des textes évoqués ne s'abandonnent pas pour autant à la contemplation stérile des surfaces associée au proverbial désespoir postmoderne. Ils échangent la transparence optimiste mais aliénante du passé contre une opacité de surface paradoxalement cristalline, car reflétant à merveille les complexités du réel. C'est ce que souligne le narrateur de London Fields par son rejet d'une licence poétique postulant la transparence irréaliste du personnage de fiction : «I'm not getting something and what I'm not getting has to do with the truth [...] In fiction (rightly so called), people become coherent and intelligible - and they aren't like that. We all know they aren't $»^{55}$.

C'est bien une certaine poétique de l'enfance qui se fait jour dans la fiction britannique contemporaine, où la transparence comme illusion de profondeur laisse place à des surfaces bombées qui bloquent toute évasion et toute élucidation facile, invitant plutôt le lecteur à une forme de responsabilité, ne serait-ce que par la remise en question des interprétations traditionnelles. Comme l'explique Riffaterre dans son analyse de l'intertextualité, le réalisme - celui qui contourne l'enfant comme corps pour substituer à la matière un sens - est bien l'autre nom de l'idéologie.

The more faithfully a text is supposed to reflect the manifold aspects of reality, or the more it proclaims itself a mirror, the more total the subordination of the mimetic multiplicity to a single message, to a semiotic oneness. [...] The literary representation of reality, then, for all its objectifying stance, is essentially an interpretive discourse. ${ }^{56}$

Plutôt, c'est un réalisme de la surface au sens de la laideur, de la non-intelligibilité du réel qui s'écrit contre celui-là, et marque au passage une angoisse toute postmoderne de perdre un peu trop de sa substance dans le monde de surfaces qu'il identifie. «I feel seemless and insubstantial, like a creation. As if someone made me up, for money " ${ }^{57}$, se plaint le narrateur de London Fields, rappelant que le prix à payer pour la libération symbolique du personnage, c'est la mise en avant de son épaisseur de papier, d'une existence en deux dimensions qui s'arrête au corps comme seul connaissable. Finalement, si le sens de l'enfant est perdu - et c'est tant mieux semblent dire les romanciers étudiés on ne peut pas en dire autant de sa capacité de signification, c'est-à-dire encore, dans sa préférence pour l'énigme et l'abject, sa capacité à venir chercher le lecteur, à le bousculer un peu.

\section{BIBLIOGRAPHIE}

Ackroyd, Peter. English Music (1992). London: Penguin, 1993. 
Amis, Martin. London Fields (1989). London: Vintage, 2003.

Atwood, Margaret. Cat's Eye (1988). London: Virago, 1990.

Barrie, J.M. Peter and Wendy (1911). New York, London, Toronto: Everyman's Library Children's Classics, 1992.

Barthes, Roland. S/Z. Paris : Seuil, 1970.

Baudrillard, Jean. Simulacres et simulation. Paris : Galilée, 1981.

Bloom, Harold. The Anxiety of Influence: A Theory of Poetry ( $2^{\text {nd }}$ edition) (1973). Oxford: OUP, 1997.

Blum, Virginia. Hide and Seek: The Child Between Psychoanalysis and Fiction. Urbana and Chicago: U of Illinois P, 1995.

Byatt, A.S. The Children's Book. London: Chatto \& Windus, 2009.

Cixous, Hélène et Clément, Catherine. La Jeune Née. Paris : Union Générale d'Éditions, 1975.

Cornis-Pop, Marcel. « Postmodernism beyond Self-Reflection: Radical Mimetism in Recent Fiction ». Mimesis in Contemporary Theory: an Interdisciplinary Approach. Vol. 2: Mimesis, Semiosis and Power. Ronald Bogue (ed.). Philadelphia and Amsterdam: John Benjamins Publishing Company, 1991, 127-155.

Dusinberre, Juliet. «The Child's Eye and the Adult's Voice: Flora Thompson's Lark Rise to Candleford ». The Review of English Studies 35.137 (Feb. 1984): 61-70.

Gonzalez, Madelena. " The Aesthetics of Post-Realism and the Obscenification of Everyday Life: The Novel in the Age of Technology ». Journal of Narrative Theory 38.1 (Winter 2008): 111-133.

Hassan, Ihab. « Pluralism in Postmodern Perspective ». Critical Inquiry 12.3 (Spring 1986): 503-520. Ishiguro, Kazuo. Never Let Me Go (2005). London: Faber and Faber, 2006.

Ishiguro, Kazuo. When We Were Orphans. New York: Vintage, 2000.

James, Henry. What Maisie Knew (1898). London: Everyman, 1997.

Kincaid, James. Child-Loving: the Erotic Child and Victorian Culture. New York and London: Routledge, 1992.

Kuhn, Reinhard. Corruption in Paradise: The Image of Childhood in Western Literature. Hanover (N.H.) and London: UP of New England, 1982.

Leavis, F.R. Foreword to Coveney, Peter. The Image of Childhood: the Individual and Society: a Study of the Theme in English Literature (1957). Harmondsworth: Penguin, 1967.

Lessing, Doris. The Fifth Child (1988). London: Harper Perennial, 2007.

McEwan, Ian. Atonement (2001). London: Vintage, 2002.

McEwan, Ian. The Cement Garden (1978). London: Vintage, 2006.

McEwan, Ian. The Child in Time (1987). London: Vintage, 1992.

Pouillon, Jean. Temps et roman (1946). Paris : Gallimard, 1993.

Riffaterre, Michael. «Intertextual Representation: On Mimesis as Interpretive Discourse ». Critical Inquiry 11.1 (Sept. 1984): 141-162.

Rousseau, Jean-Jacques. Discours sur les sciences et les arts (1750). Paris : Garnier Flammarion, 1971. Rousseau, Jean-Jacques. Émile ou de l'éducation (1762). Paris : Garnier Flammarion, 1966. 
Starobinski, Jean. Jean-Jacques Rousseau : la transparence et l'obstacle. Paris : Gallimard, 1971.

\section{NOTES}

1. Hélène Cixous et Catherine Clément, La Jeune Née, Paris : Union Générale d'Éditions, 1975, 53.

2. Par exemple : Jean-Jacques Rousseau, Discours sur les sciences et les arts (1750), Paris : Garnier Flammarion, 1971, 40.

3. Kazuo Ishiguro, When We Were Orphans, New York: Vintage, 2000, 8-9.

4. Jean-Jacques Rousseau, Émile ou de l'éducation (1762), Paris : Garnier Flammarion, 1966, 74.

5. Henry James, What Maisie Knew (1898), London: Everyman, 1997.

6. Juliet Dusinberre, "The Child's Eye and the Adult's Voice: Flora Thompson's Lark Rise to Candleford ", The Review of English Studies 35.137 (Feb. 1984), 61-70, 70.

7. Reinhard Kuhn, Corruption in Paradise: The Image of Childhood in Western Literature, Hanover (N.H.) and London: UP of New England, 1982, 21.

8. James, op. cit., 216.

9. Virginia Blum, Hide and Seek: The Child Between Psychonanalysis and Fiction, Urbana and Chicago: U of Illinois P, 1995, 6 et 8.

10. Ibid., 248.

11. Pas de langage, mais plutôt, dans les mots de Starobinski analysant Rousseau, la «transparence réciproque des consciences» (Jean Starobinski, Jean-Jacques Rousseau: la transparence et l'obstacle, Paris : Gallimard, 1971, 19).

12. J.M. Barrie, Peter and Wendy (1911), New York, London, Toronto: Everyman's Library Children's Classics, 1992.

13. Blum, op. cit., 248.

14. A.S. Byatt, The Children's Book, London: Chatto \& Windus, 2009, 81.

15. Rousseau, Émile ou de l'éducation, op. cit., 112-113.

16. Byatt, op. cit., 473.

17. Ian McEwan, The Child in Time (1987), London: Vintage, 1992, 2.

18. Ibid., 61.

19. James Kincaid, Child-Loving: the Erotic Child and Victorian Culture, New York and London: Routledge, 1992, 306 et passim.

20. Vladimir Nabokov, Lolita (1955), London: Penguin, 2006.

21. Martin Amis, London Fields (1989), London: Vintage, 2003, 210.

22. Byatt, op. cit., 279.

23. Kuhn, op. cit., 20.

24. Doris Lessing, The Fifth Child (1988), London: Harper Perennial, 2007, 140.

25. Amis, op. cit., 155.

26. Roland Barthes, S/Z, Paris : Seuil, 1970, 61, 87 et passim.

27. Michael Riffaterre, «Intertextual Representation: On Mimesis as Interpretive Discourse », Critical Inquiry 11.1 (Sept. 1984): 141-162.

28. Peter Ackroyd, English Music (1992), London: Penguin, 1993, 12.

29. Ibid., 87. « He could not have existed without me, I believe, but he does not belong to me. »

30. Ibid., 92.

31. Harold Bloom, The Anxiety of Influence: A Theory of Poetry ( $2^{\text {nd }}$ edition), 1973, Oxford: OUP, 1997.

32. Riffaterre, op. cit., 158. Il met en garde contre : « the lure of the referential fallacy ».

33. Amis, op. cit., 181-182 et McEwan, op.cit., 128.

34. Jean Pouillon, Temps et roman (1946), Paris : Gallimard, 1993, 71.

35. Ian McEwan, Atonement (2001), London: Vintage, 2002. 
36. Ihab Hassan, «Pluralism in Postmodern Perspective », Critical Inquiry 12.3 (Spring 1986), 503-520, 507.

37. Marcel Cornis-Pop, « Postmodernism beyond Self-Reflection: Radical Mimetism in Recent Fiction ", Mimesis in Contemporary Theory: an Interdisciplinary Approach, Vol. 2: Mimesis, Semiosis and Power, Ronald Bogue (ed.), Philadelphia and Amsterdam: John Benjamins Publishing Company, 1991, 127-155, 130.

38. Amis, op. cit., 223.

39. Amis, op. cit., 363.

40. Madelena Gonzalez, "The Aesthetics of Post-Realism and the Obscenification of Everyday Life: The Novel in the Age of Technology ", Journal of Narrative Theory 38.1 (Winter 2008), 111-133, 113. «[...] contemporary fiction [...] is likewise characterized by an obsession with overexposure. [...] the ordinary and the quotidian endlessly contemplated take on the lurid detail of the pornographic ».

41. Amis, op. cit., 140.

42. Ian McEwan, The Cement Garden (1978), London: Vintage, 2006.

43. Gonzalez, op. cit., 121.

44. Lessing, op. cit., 98.

45. F.R. Leavis, Foreword to Peter Coveney, The Image of Childhood: the Individual and Society: a Study of the Theme in English Literature (1957), Harmondsworth: Penguin, 1967, 23.

46. Byatt, op. cit., 7.

47. Ibid., 11.

48. Kazuo Ishiguro, Never Let Me Go (2005), London: Faber and Faber, 2006.

49. Amis, op. cit., 212-213.

50. Hassan, op. cit., 506.

51. Jean Baudrillard, Simulacres et simulation, Paris : Galilée, 1981.

52. Ibid., 17, 33 et passim.

53. Byatt, op. cit., 505-506.

54. Ibid., 506.

55. Amis, op. cit., 240.

56. Rifaterre, op. cit., 159.

57. Amis, op. cit., 470.

\section{RÉSUMÉS}

Chercher l'enfant dans le roman britannique contemporain, c'est souvent s'exposer au paradoxe de son omniprésence dans les discours, conjuguée à une forme d'absence physique. Dans The Child in Time, de McEwan, il disparaît avant même la diégèse pour apparaître chez Lessing ou Martin Amis sous la forme creuse du changeling. Dans When We Were Orphans, Ishiguro dessine un enfant voilé par les complaisances fallacieuses du souvenir, tandis que chez Byatt (The Children's Book) les protagonistes se voient imposer par leur mère-écrivain un double fictionnel qui les déréalise. C'est cette singulière présence-absence, cette très légère façon qu'a l'enfant de se poser en littérature, qui semble avoir fasciné les romanciers contemporains, en ce que, objet littéraire non verbal (infans), et toujours l'autre de l'adulte qui l'écrit, il est d'abord un défi à la transparence mimétique, contraignant ceux qui l'écrivent à exhiber leurs filtres, souvent à 
travers une thématique assumée du faux, du leurre. La seconde transparence de l'enfant n'est pas strictement poïétique mais tient à sa représentation : défini par le déjà-là littéraire comme un idéal par l'absence (innocent, infans...), il attire l'investissement aliénant de toutes les valeurs jusqu'à devenir un signe blanc, une opacité herméneutique que certains romanciers cherchent aujourd'hui à conjurer en salissant l'enfant, mon(s)trant son corps pour mieux lui rendre l'ombre portée qui lui manquait. Une autre manière de travailler cet héritage spectral étant de laisser voir, en transparence, l'épaisseur feuilletée de palimpseste de l'enfant: plutôt qu'un corps, d'aucuns choisissent d'exhiber sans la résoudre la cacophonie des discours et symboles par des références explicites ou non aux hypotextes et métarécits de l'enfance, faisant du vide brouillé un jeu de lumières.

Looking for the figure of the child in contemporary British fiction, one is confronted with the paradox of its omnipresence in discourse, matched only by an equally ostentatious physical evanescence. In McEwan's The Child in Time, the child of the title is gone before the narrative has even begun, while Ishiguro's When We Were Orphans veils it in confusing layers of fallacious memories, and Byatt's The Children's Book stages the slow shedding of its young protagonists' substance, as their mother gradually turns them into a work of fiction. This very particular kind of absence-cum-presence, which imbues the child with a unique lightness of being, seems to have mesmerized contemporary writers. Exploiting the poetic resources of the strangely non-verbal ( infans) literary tool, they strive to prise open a figure so alien to the grown-up writer, and one that is such a challenge to transparency, that it invariably leads them to write instead about the impossibility of mimetic art, about fakes, about trompe-l'oeil fiction. For all its apparent poetic opacity, the child is indeed transparent in more ways than one: defined by centuries of literature as an ideally blank space of non-being, the innocent infant has become a repository for all adult values, a self-cancelling sign both opaque in meaning and see-through in substance which several contemporary novelists are now trying to liberate from semiotic alienation by sullying it. To have a visible body again, the child must become a poetic monster; for Peter to finally cast a shadow, the chimerical nature of its body - a composite of alien symbolic and discursive fragments - must be exhibited for the palimpsestic mess that it is.

\section{INDEX}

Mots-clés : enfant, roman contemporain, transparence, poétique, aliénation sémiotique

Keywords : child, contemporary fiction, transparency, poetics, semiotic alienation

oeuvrecitee Child in Time (The), Children's Book (The), English Music, Fifth Child (The), London Fields, When We Were Orphans

\section{AUTEURS}

\section{CAMILLE FRANÇOIS}

Ancienne élève de l'École Normale Supérieure et agrégée d'anglais, Camille François-Paulos termine actuellement une thèse de littérature anglaise sous la direction de Camille Fort à l'Université de Picardie, et enseigne parallèlement à l'Université de Savoie. Ses recherches portent sur l'écriture de l'enfant dans le roman britannique contemporain, et c'est au travers du double prisme de la narratologie et des cultural studies qu'elle examine cette question, principalement dans l'œuvre de A.S. Byatt, Ian McEwan, Martin Amis, Kazuo Ishiguro et Doris Lessing. Elle a publié plusieurs articles en français et en anglais sur le sujet, et s'intéresse particulièrement à l'association traditionnelle de l'enfant avec les idées de perception, 
d'imagination, et la figure du poète, à la représentation du corps de l'enfant, ou encore à la manière dont la présence de l'enfant dans un texte en détermine la structure éthique et esthétique. 